\title{
OPTIMIZATION TECHNIQUES FOR HARMONICS MINIMIZATION IN CASCADED HYBRID MULTILEVEL CONVERTERS: A REVIEW
}

\author{
Parul Gaur ${ }^{1}$, Preeti Singh ${ }^{2}$ \\ ${ }^{I}$ Assistant Professor, Electrical and Electronics Engineering, UIET, Chandigarh, India \\ ${ }^{2}$ Assistant Professor, Electronics and Communication Engineering, UIET, Chandigarh, India
}

\begin{abstract}
Recent advancements in the field of power electronics have made possible the worldwide application of converters in dc power source utilization, uninterruptible power supplies, electric motor speed control, switched mode power supplies, flexible alternating current transmission systems devices including static synchronous compensator and their controls. Power semiconductor devices such as converters have been emerged as powerful devices in the power industries. As such, harmonics free output of inverters is assuming extreme importance. Over the past decades, depending upon the topologies and control strategies, numerous optimization techniques have been proposed for desired output waveform. This paper presents a review of optimization techniques used for multilevel inverters. The pros and cons of optimization techniques are discussed. The objective of these optimization techniques is to find out the optimum firing angles of multilevel inverters, which results in minimum harmonics. As a preferred option for proposed work, reduction of total harmonic distortion with the aid of particle swarm optimization technique to multilevel inverters is suggested.
\end{abstract}

Keywords: Power Converters, Multilevel Inverters (MLI), Cascaded Hybrid Bridge (CHB), Modulation, Switching Frequency, Harmonic Distortions.

\section{INTRODUCTION}

Ever since the advent of thyristors in mid 1960s, the field of power electronics has become very popular in almost all industrial applications; whether it may be electrical, electronics, chemical, aerospace, textile etc. [1-3]. Power converters have taken a very important place in the industrial world. Initially the scope was limited up to two level converters but nowadays multilevel converters such as three levels, five levels and higher levels converters have been designed depending upon the various topologies. The main concern of system designers and application engineers has been the task of appropriate designing of multilevel converters which produce desired staircase wave with fewer harmonics. This has been mainly due to the reason that on increasing the number of levels, more harmonics are introduced in the output of the inverters. The multilevel inverter having harmonics free output attracted importance in all industrial, commercial, domestic and defense applications [4]. Hence, there has arisen the need for suitable control strategies and optimization techniques to achieve harmonics free output in multilevel inverters [5-7].

Neutral point clamped (NPC), flying capacitor (FC) and cascaded hybrid bridge (CHB) are the three multilevel converter topologies and well documented in the literature [8]. A proper selection of topology using power semiconductor devices forms the most ideal inverter for a variety of industrial applications. More emphasis will be given here to the features related to the $\mathrm{CHB}$, since it is the topology to be used in this paper for harmonic control. In the late 1960s, with the series connected hybrid bridge multilevel stepped waveform concept, CHB topology came into existence [9]. Compared with NPC and FC topologies, cascaded hybrid bridge converters require the minimum number of components for producing the same number of voltage levels because of elimination of clamping diodes and voltage balancing capacitors. But $\mathrm{CHB}$ inverter needs separate dc sources for each cell. After selection of multilevel inverter topology; there is need to decide the control or modulation strategies and optimization techniques, which result in minimum total harmonic distortion (THD) as mentioned in standards like IEEE-519, EN 50160, IEC 61000-2-2, IEEE 61000-2-4 etc. [10].

This paper is arranged into six sections. Section I deals with the introduction, gives the brief history of multilevel inverter topologies, control strategies and optimization techniques. Most widely used CHB topology is described in section II. Suitable modulation techniques for designing of multilevel inverters are mentioned in section III. Section IV enlists the optimization techniques for finding the optimum firing angles. Comparison of all the existing optimization techniques has done in this section. Challenges and future scope are mentioned in section V. Finally conclusion has drawn in section VI.

\section{CASCADED HYBRID MULTILEVEL TOPOLOGY}

Cascaded hybrid multilevel is an important topology of multilevel inverters because of various advantages [11]. This topology includes the series of H-bridge inverter units with separate dc sources for each unit. A n level topology 
requires 2(n-1) switches and (n-1)/2 isolated power supplies per phase. Fig. 1 shows one leg of 7 level CHB inverter. Three different voltage levels are produced by each $\mathrm{H}-$ bridge, say bridge $\mathrm{H} 1$ will produce: $+\mathrm{Vdc}, 0$ and $-\mathrm{Vdc}$ voltages by different switching patterns of four switches i.e. $\mathrm{S} 1, \mathrm{~S} 2, \mathrm{~S} 3$ and S4. In the same manner, other bridges also produce the voltages. Each H-bridge produces the ac output. The synthesized output voltage waveform is sum of all individual H-bridges voltages.

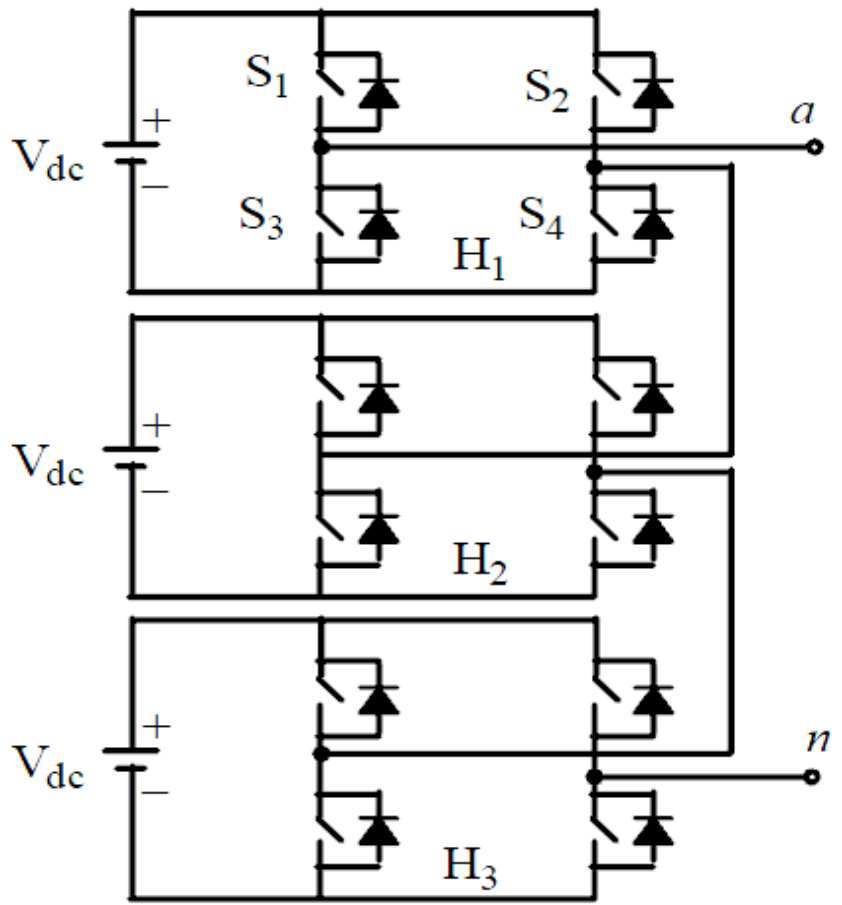

Fig-1: Configuration of Seven Level Cascaded Inverter

The sinusoidal output voltage produced by cascaded multilevel inverter depends upon the firing angles of switching devices used in H-bridge. Switching devices of each $\mathrm{H}$-bridge are fired at different angles. For e.g. in 7 level $\mathrm{CHB}$ inverter, three bridges i.e. $\mathrm{H} 1, \mathrm{H} 2$ and $\mathrm{H} 3$ are fired at three different angles $\alpha 1, \alpha 2$ and $\alpha 3$ respectively. Firing angles value are selected in such a way so that the inverter produces the harmonics free sinusoidal waveform.

Fig. 2 shows the output waveform for 7 level MLI. As the output voltage generated by cascaded MLI depends on these firing angles, the output voltage needs to vary from zero to maximum (corresponding variation in modulation index is from 0 to 1). Generally, these firing angles are calculated with the aid of optimization techniques and are stored in a look up table and accordingly switching devices are fired.

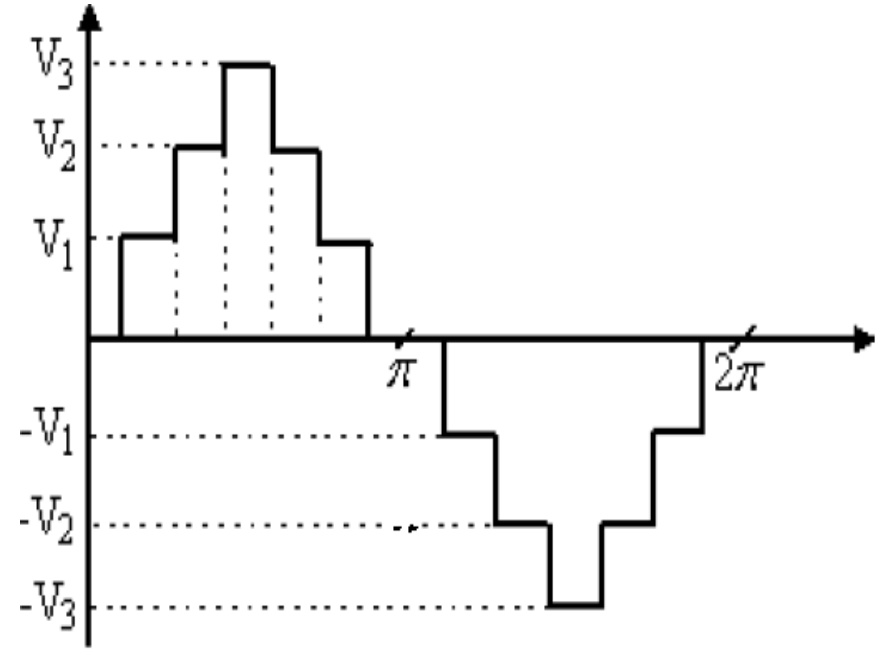

Fig-2: Typical Output Voltage Produced by Seven Level Cascaded Inverter

\section{MULTILEVEL INVERTER CONTROL STRATEGIES}

Another important feature of MLI is the various control strategies applied to it [12]. The objective of these modulation techniques is to get a switched stepped waveform with fewer harmonics and losses. Fig. 3 shows the classification of modulation techniques based on the switching frequency.

Numerous pulse width modulation (PWM) and space vector control (SVC) strategies, which result in less complexity and better output waveform have been reported in the literature [13]. High power applications require fundamental or low switching frequency modulation techniques to keep the losses below the acceptable values. Another option is selective harmonic control (SHC) technique. SHC strategy includes selective harmonic elimination (SHE) and selective harmonic mitigation (SHM). SHE technique is also known as fundamental switching frequency method as per the harmonic elimination theory proposed by Patel et al [14, 15],. In this control strategy, firing angles are selected such that the THD is minimum in the output voltage waveform.

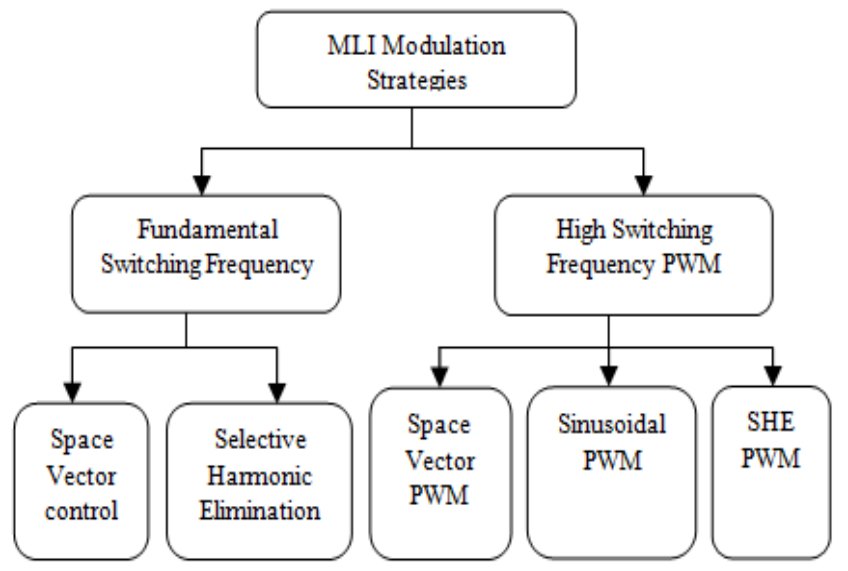

Fig-3: Classification of Modulation Techniques 


\section{OPTIMIZATION TECHNIQUES}

In MLIs, output voltage is represented using fourier series as:

$$
V_{a n}(w t)=\sum_{m=1,3,5, \ldots .}^{\infty} \frac{4 V_{d c}}{m \Pi}\left(\cos \left(\mathrm{m} \alpha_{1}\right)+\cos \left(\mathrm{m} \alpha_{2}\right)+\ldots \ldots \ldots \ldots . .+\cos \left(\mathrm{m} \alpha_{n}\right)\right) \sin (\mathrm{m} w t)
$$

Where $V_{d c}=$ dc voltage and $0 \leq \alpha 1<\alpha 2<\alpha 3 \leq \Pi / 2$

The harmonic factor (percentage) of the nth harmonic is calculated as:

$$
H F_{n}=\frac{V_{n}}{V_{1}} \mathrm{X} 100 ; n>1
$$

Here $V_{n}$ represents the $\mathrm{n}^{\text {th }}$ harmonic voltage and $V_{1}$ is fundamental output voltage.

Equation (i) can be divided into three parts as:

$V_{a n}(w t)=V_{l 1}(\mathrm{t})+V_{l 2}(\mathrm{t})+V_{l 3}(\mathrm{t})$

Where $V_{l 1}(t)$ is the fundamental frequency voltage, represented as:

$V_{l 1}(t)=\frac{4 V_{d c}}{\Pi}\left(\cos \alpha_{1}+\cos \alpha_{2}+\cos \alpha_{3}\right) \sin w t \quad[$ For 7 level cascaded MLI]

$V_{l 2}(t)$ is the triplen harmonic voltages as:

$V_{l 2}(t)=\sum_{m=3,9,15, \ldots . .} \frac{4 V_{d c}}{m \Pi}\left(\cos \left(m \alpha_{1}\right)+\cos \left(m \alpha_{2}\right)+\cos \left(m \alpha_{3}\right)\right) \sin (m w t)$

$V_{l 3}(t)$ is the odd harmonic (except triplen) voltages as:

$V_{l 3}(t)=\sum_{m=5,7,11, \ldots \ldots . .} \frac{4 V_{d c}}{m \Pi}\left(\cos \left(m \alpha_{1}\right)+\cos \left(m \alpha_{2}\right)+\cos \left(m \alpha_{3}\right)\right) \sin (m w t)$

In three phase applications, triplen harmonic voltages in each phase cancel out automatically, hence no need to cancel these voltages. Another important parameter is modulation index $\left(\mathrm{m}_{\mathrm{I}}\right)$, which represents the relationship between the fundamental voltage $\left(\mathrm{V}_{1}\right)$ and the maximum obtainable voltage $\left(\mathrm{V}_{1 \text { max }}\right)$. It is defined as the ratio of the fundamental output voltage to the maximum obtainable fundamental voltage. Switching angles $\alpha_{1}, \alpha_{2}$ and $\alpha_{3}$ (in case of 7 level cascaded MLI) can be found using optimization techniques.

Equation (i) is known as non linear transcendental equations. For solving these transcendental equations, different optimization techniques have been suggested in the literature. In [16], a method was suggested so as to produce the required output voltage and simultaneously to suppress the higher order harmonics. The transcendental equations involving the harmonic content are converted into polynomial equations. These equations are further solved by the method of resultants. But in this technique, the degree of polynomials become quite large when there are numerous dc sources, which further results in high computational burden of resultant polynomials. Also, due to the computational complexity associated with these techniques, theory of resultant and symmetrical polynomials has been applied up to 11 level multilevel converter only. Limitation of resultant theory appears when applied to MLIs with unequal dc sources, where transcendental equations are no longer symmetrical and requires the solution of a set of higher degree equations.

In $[17,18]$ switching angles are calculated using Newton Raphson (N-R) numerical technique, where certain number of harmonic components have eliminated. But N-R methods have some drawbacks like divergence problems, need to define initial value and also provides no optimum solution.

Genetic algorithm (GA) technique is used in [19] for eliminating some higher order harmonics while maintaining the required fundamental voltage. GA is a computational approach by which optimization problems can be solved using genetic methods and the theory of evolution. But for implementation of this method, proper selection of certain parameters such as initial population size, crossover operation, mutation operation etc. are required, thereby implementation of this algorithm becomes tedious for higher MLIS.

In $[20,21]$, a new approach has discussed for real time calculation of firing angles using artificial neural networks (ANN). The approach is accomplished by first transforming the nonlinear transcendental harmonic elimination equations for all possible switching schemes into a one input (modulation index) and multi output (switching angles) three layers ANN. Then, the complete set of solutions of the equations is found using the back propagation of the errors between the desired harmonic elimination and the non linear equation systems output using the switching angle given by the ANN. Simulations in the [21] indicates that the switching angles issued by look up table and through trained neural network are almost equal. Therefore, a conclusion has drawn that a look up table can be replaced by a trained neural network, hence reducing the computational effort and storing capacity. Further a trained neural network produces switching angles by interpolation/extrapolation even for those values of modulation index, where switching angles are not calculated.

In [22], generalized pattern search (GPS), simulated annealing (SA) and genetic algorithm (GA) are used for calculating the firing angles to eliminate harmonics in 13 level inverter. The proposed algorithms can be applied to higher MLIs. The simulation results showed that GPS and SA methods are more efficient than GA. 
Real time calculation of switching angles for minimum THD has done using step modulation [23]. However, the limitation of stepped modulation technique lies in its narrow modulation index. Bee optimization technique is used in [24] for harmonic elimination in cascaded MLI. In this paper, 7 level cascaded MLI is used. The algorithm is based on food foraging behaviour of a swarm of a honeybees. Simulation results showed that bee algorithm (BA) has higher precision and probability of convergence than GA.

Harmony search algorithm (HSA) and particle swarm optimization (PSO) are other optimization techniques for finding out the firing angles of cascaded MLIs [25]. HSA searches those certain values which optimize the fitness function and also simultaneously satisfy the problem's constraints. HSA imposes fewer mathematical requirements and does not require initial value settings for decision variable. For optimization of non linear transcendental equations, PSO methodology is a very powerful approach. In [26], a novel PSO technique to determine the optimum firing angles of MLIs is presented. This optimization technique is applied to non linear transcendental equations characterizing the harmonic content to minimize the low order harmonics. Figure (iv) shows the flowchart of PSO technique. Simulation results showed that PSO can simply find the optimum switching angles and has faster convergence with better quality solutions than GA approach. PSO completely outperforms both GA and HSA. [27] presents PSO based optimal switching technique for harmonic elimination in cascaded MLIs.

A species based PSO (SPSO) method, which includes the suitable adjustment of niche radius for calculation of the optimum firing angles of MLIs, has been proposed in [28]. Simulation and hardware results are mentioned for cascaded hybrid 11 level inverter. Results indicate that all the lower as well as higher order harmonics are effectively minimized in the output sinusoidal voltage waveform of MLI. Also the switching frequency of multilevel inverter and the THD have decreased dramatically.

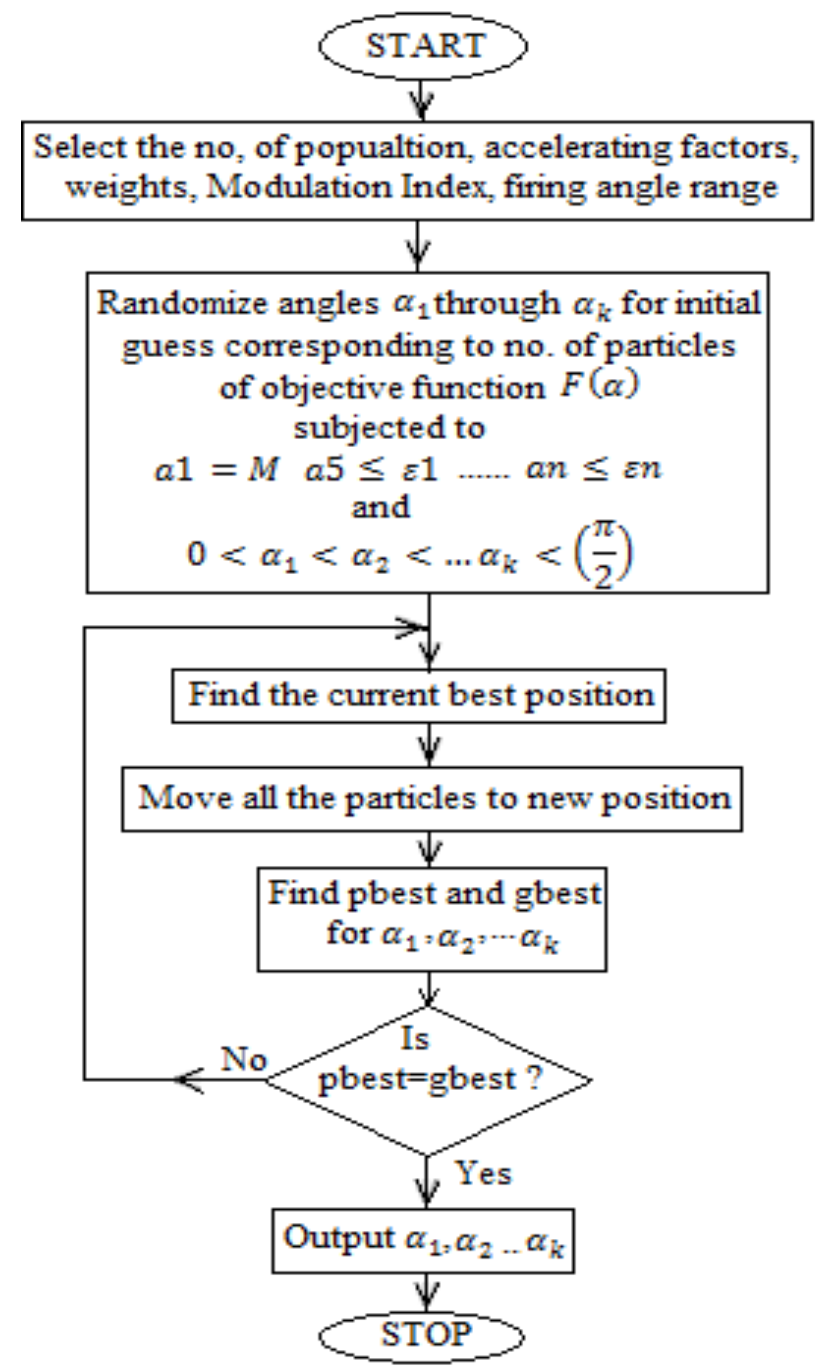

Fig-4: Flowchart of Particle Swarm Optimization Algorithm

\section{CHALLENGES AND FUTURE SCOPE}

Numerous harmonics optimization techniques for cascaded MLIs have been reported in the literature but still there are some challenges which need to be overcome. Some of these challenges are discussed in the succeeding paragraphs.

In cascaded MLIs, with increase in levels output voltage waveform tends to become more sinusoidal but also leads to more harmonics. Optimization techniques eliminate or minimized the lower order harmonics; still there exist some higher order harmonics. For minimizing these higher order harmonics use of active filters are necessary, which ultimately leads to overall increase in cost of MLI [29]. However, some optimization techniques minimize the higher order harmonics too; but those techniques have computational burden and are time consuming. In this context, selection of suitable optimization technique is an important consideration while designing the cascaded MLIs. For a particular value of modulation index, multiple set of solutions of non linear transcendental equations exists. The main challenge is to select that solution, which results in minimum THD of output voltage waveform of cascaded MLI. 
In optimization techniques, no solution exists for a particular value of modulation index. However, in the literature optimization techniques have been reported which provide a solution set for complete range of modulation index [30]. Even after lots of advancement in harmonic optimization techniques, there is further scope to reduce the THD of output voltage waveform by selection of suitable optimization technique while maintaining the permissible switching frequencies.

\section{CONCLUSION}

The different optimization techniques such as Newton Raphson method, resultant theory and symmetric polynomial, genetic algorithm, harmony search algorithm, particle swarm optimization etc. have been proposed to minimize the total harmonic distortion in cascaded multilevel inverters. Maintaining the desired level of fundamental output voltage, all the lower order harmonics are minimized or controlled within the permissible limits. Thereby, results in minimum total harmonic distortion and the corresponding firing angles are determined. The proposed methods are able to find the optimum firing angles in a simple manner. These techniques ensure the accuracy and quality of firing angles of cascaded multilevel inverters such that output voltage waveform results in minimum total harmonic distortion.

\section{REFERENCES}

[1]. M.M. Ektessabi, C.M. Bhatia, S.S. Lamba; "A Dedicated Digital Implementation of PWM Inverters", IASTED International Conference, July $8-10$, 1992, University of Tehran, Iran.

[2]. C. M. Bhatia; "Design Development and Fabrication of MA 818 Controller for Three- Phase Voltage Source Transistorised PWM Inverter", Research Monograph Number IDESP/MAP/02/1993; Microprocessor Application Programme of IIT Delhi, 1993.

[3]. C. M. Bhatia, M. S. Rao, Ali Salem Bathuniya, Pradeep Kumar, "Design Development and Fabrication of MA 818 controller for a Three-phase, 10 KVA, Voltage Source Transistorized PWM Inverter", Journal of IETE, Vol. 11, pp. 341-346, Sept- Dec. 1994.

[4]. S. Bernet, "Recent developments of high power converters for industry and traction applications," IEEE Transactions on Power Electronics, vol. 15, no. 6. pp. 11021117, Nov. 2010.

[5]. J. Rodriguez, J.S. Lai and F.Z. Peng, " Multilevel inverters: A survey of topologies, controls, and applications," IEEE Transactions on Industrial Electronics, vol. 49, no. 4. pp. 724-738, Aug. 2002.

[6]. J. Rodriguez, S. Bernet, B. Wu, J. O. Pontt, and S. Kouro, "Multilevel voltage source converter topologies for industrial medium voltage drives," IEEE Transactions on Industrial Electronics, vol. 54, no. 6. pp. 2930-2945, Dec. 2007.

[7]. S. S. Fazel, S. Bernet, D. Krug, and K. Jalili, "Design and comparison of neutral point clamped, flying capacitors, and series connected H-bridge multilevel converters," IEEE
Transactions on Industrial Electronics, vol. 43, no. 4. pp. 1032-1040, Aug. 2007.

[8]. J. Rodriguez, J.Holtz, H .A. Rub, and G. Baoming, "Medium voltage multilevel converters-state of the art, challenges and requirements in the industrial applications," IEEE Transactions on Industrial Electronics, vol. 57, no. 8. pp. 2581-2596, Aug. 2010.

[9]. J.S. Lai and F.Z. Peng, "Multilevel converters-a new breed of power converters," IEEE Transactions on Industrial Electronics, vol. 32, no. 3. pp. 509-517, May 1996.

[10]. Z. Pan, F.Z. Peng, K.A. Corzine, V.R. Stefanovic, J.M. Leuthen, and S. Gataric, "Voltage balancing control of diode clamped multilevel rectifier/inverter systems," IEEE Transactions on Industrial Electronics, vol. 41, no.6. pp. 1698-1706, Nov. 2005.

[11]. F.Z.Peng, J.W.McKeever and D.J.Adams, "Cascade multilevel inverters for utility applications," IECON Proceedings (Industrial Electronics Conference), vol.2, pp. 437-442, 1997.

[12]. Parul Gaur and Preeti Singh, "Various control strategies for medium voltage high power multilevel converters: A review," IEEE conference RAECS-2014 held at UIET, Chandigarh, India, 6th-8th March, 2014, pp. 1-6.

[13]. L.Ben-Brahim, "A discontinuous PWM method for balancing the neutral point voltage in three level inverter fed variable frequency drives," IEEE Transactions on Energy Converters, vol. 23, no.4. pp. 1057-1063, Dec. 2008.

[14]. L.G. Franquelo, J. Napoles, R.C. Guisado, J.I. Leon, and M. Aguirre, "A flexible selective harmonic mitigation technique to meet grid codes in three level PWM converters," IEEE Transactions on Industrial Electronics, vol. 54, no.6. pp. 3022-3029, Dec. 2007.

[15]. S.R.Pulikanti, Georgios Konstantinou, and Vassilios G. Agelidis, "Hybrid seven level cascaded active neutral point clamped based multilevel converter under SHEPWM," IEEE Transactions on Industrial Electronics, vol. 60, no. 11. pp. 4794-4804, Nov. 2013.

[16]. J.N. Chaisson, L.M. Tolbert, K.J. Mckenzine and Zhong Du, "Elimination of harmonics in a multilevel converter using the theory of symmetric polynomials and resultants," IEEE transactions on Control Systems Technology, vol. 13, no. 2, March 2005.

[17]. Jagdish Kumar, "THD analysis for different levels of cascade multilevel inverters for industrial applications," International Journal of Emerging Technology and Advanced Engineering, vol. 2, issue 10, Oct 2012.

[18]. L.M. Tolbert, F.Z. Peng and T.G. Habetler, "Multilevel converters for large electric drives," IEEE Transactions on Industrial Applications, vol. 35, no. 1, pp. 36-44, Feb 1999. [19]. B. Ozpineci, L.M. Tolbert and J.N. Chaisson, "Harmonic optimization of multilevel converters using genetic algorithms," 35th Annual IEEE Power Electronics Specialists Conference, Germany 2004.

[20]. O. Bouhali, F. Bouaziz, N. Rizoug and A. Talha, "Solving harmonic elimination equations in multilevel inverters by using neural networks," International Journal of Information and Electronics Engineering, vol. 3, no.2 pp. 191-195, March 2013.

[21]. Jagdish Kumar, Jaimala Gambhir and Anil Kumar, "Control of switching angles for a cascade multilevel 
inverter using ANN," IEEE conference RAECS-2014 held at UIET, Chandigarh, India, 6th-8th March, 2014, pp. 1-6.

[22]. K. Haghdar, H.A. Shayanfar and M.H.S. Alavi, "Selective harmonic elimination of multilevel inverters via methods of GPS, SA and GA," Proceedings of the 2011 Asia-Pacific Power and Energy Engineering Conference, pp. 1-5, Jan 2011.

[23]. Y. Liu, H. Hong and A.Q. Huang, "Real time calculation of switching angles minimizing THD for multilevel inverters with step modulation," IEEE Transactions on Industrial Electronics, vol. 56, no. 2, pp. 285-293, Feb 2009.

[24]. A. Kavousi, B. Vahidi, R.Salehi et al, "Application of the bee algorithm for selective harmonic elimination strategy in multilevel inverters," IEEE Transactions on Power Electronics, vol. 27, no. 4, pp. 1689-1696, April 2012.

[25]. Suman Debnath, R.N. Ray and T. Ghosh, "Comparison of different soft techniques applicable to multilevel inverter for harmonic elimination," International Journal of Computer Application, issue 2, vol. 6, Dec 2012.

[26]. H. Taghizadeh and M.T. Hagh, "Harmonic elimination of cascade multilevel inverters with non equal dc sources using particle swarm optimization," IEEE Transactions on Industrial Electronics, vol. 57, issue 11, pp. 3678-3684, Nov 2010.

[27]. R.N. Ray, D. Chatterjee and S.K. Goswami, "A particle swarm optimization based optimal switching technique for voltage harmonic reduction of multilevel inverter," Expert Systems with Applications, Elsevier, vol. 37, issue 12, pp. 7796-7801, Dec 2010.

[28]. M.T. Hagh, H. Taghizadeh and K. Razi, "Harmonic minimization in multilevel inverters using modified species based particle swarm optimization," IEEE Transactions on Power Electronics, vol. 24, issue 10, pp. 2259-2267, Oct 2009.

[29]. Zhong Du, L.M. Tolbert, J.N. Chaisson and B. Ozpineci, "Reduced switching frequency active harmonic elimination for multilevel inverters," IEEE Transactions on Industrial Electronics, vol. 55, no. 4, pp. 1761-1770, April 2008.

[30]. L.M. Tolbert, Jianze Wang, Yanchao Ji et al, "Elimination of harmonics in a modular multilevel converter using particle swarm optimization based staircase modulation strategy," IEEE Transactions on Industrial Electronics, vol. 61, no. 10, pp. 5311-5322, May 2014. 